\title{
Working out with
} weed

\section{Scientists investigate the counter-intuitive connections between exercise and cannabis.}

\section{BY TIEN NGUYEN}

$\mathrm{T}$ The stereotypical image of a cannabis smoker is someone who sprawls on the sofa for hours surrounded by a haze of smoke and half-eaten snacks. The scene is played up for laughs in films, but social psychologist Angela Bryan thought it could be cause for concern. After all, cannabis is known to increase appetite and aid relaxation, which might put people at risk of health conditions such as obesity, says Bryan, who is at the University of Colorado Boulder.

But digging into health trends revealed the opposite. Nationwide US studies report that, compared to non-users, cannabis users actually have a lower prevalence of obesity ${ }^{1}$. Intrigued, she began to investigate. Earlier this year, her team surveyed more than 600 cannabis users living in US states where the drug is legal about their exercise habits, among other health factors $^{2}$. Four out of five respondents said that they use marijuana right before or after exercising. And those users spent more minutes per week exercising than users who didn't mix the two. "We were shocked," Bryan says.

Her findings and those of others suggest that using cannabis before or after working out could be common. But scientists know very little about the effects cannabis could have on exercise. A handful of studies were conducted decades ago, but since then laboratories in the United States have found it difficult to run controlled cannabis studies because of federal restrictions. Instead, researchers are turning to surveys and anecdotal reports to piece together the biological mechanisms by which cannabis might affect physical activity.

\section{A SURVEY SAYS}

In Bryan's survey, about $70 \%$ of respondents who used cannabis before working out said

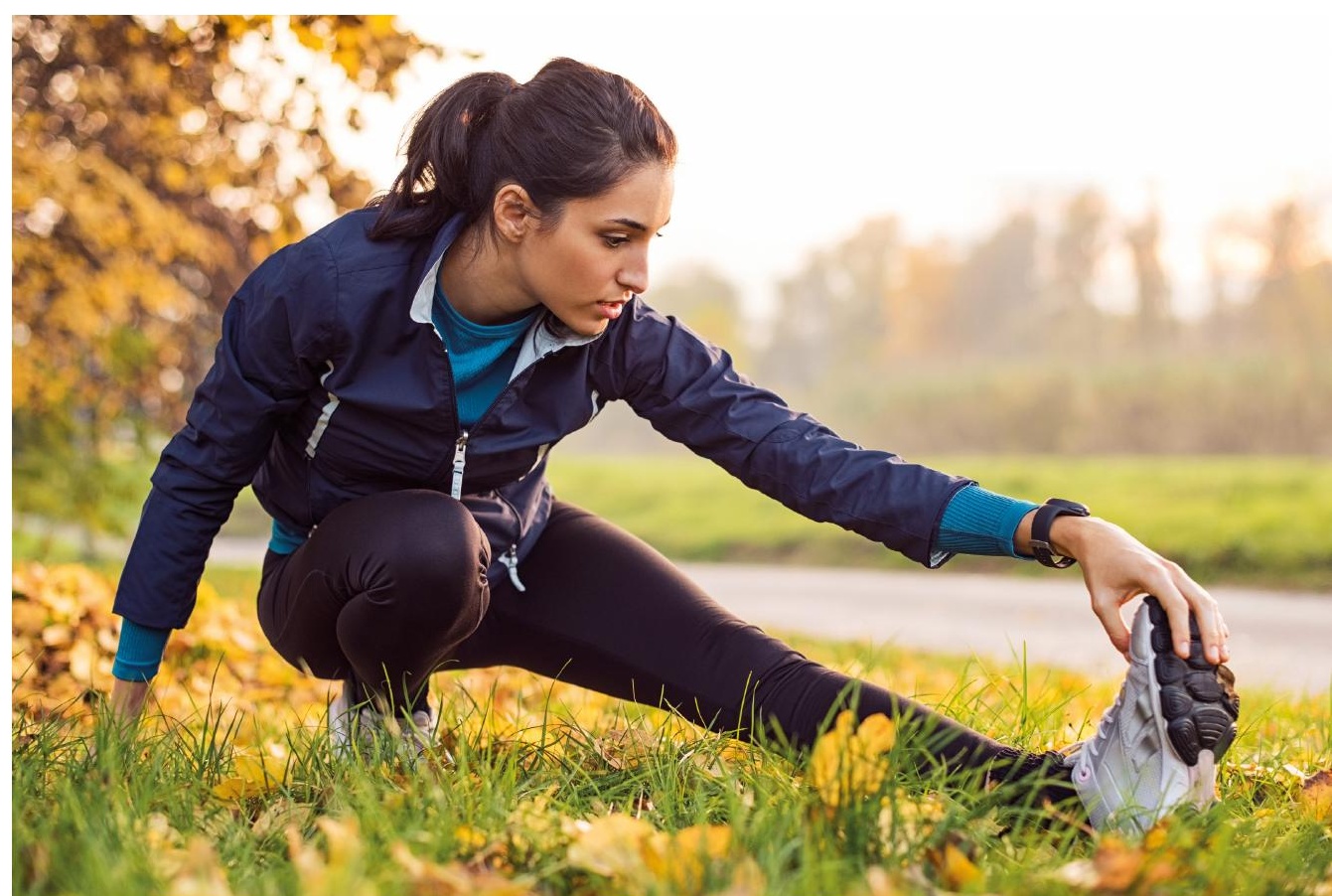

Some sportspeople claim that using cannabis enhances their athletic performance.

doing so made exercising more enjoyable. People who use cannabis might say that taking the drug makes any activity more fun, but Bryan suggests that in the case of exercise there are specific chemical interactions at play.

Consider a runner's high, the feeling of euphoria that kicks in when some people reach a sweet spot in their workout. The experience has been attributed to the release of chemicals in the brain called endorphins, but solid evidence of this is wanting. For instance, endorphins are thought to give a pleasurable feeling because they activate opioid receptors. But researchers have found that people who take opioid-blocking drugs before exercising can still achieve states of bliss during a workout ${ }^{3}$.

An alternative suggestion is that exerciseinduced euphoria originates in the endocannabinoid system. A 2003 study $^{4}$ found elevated levels of the endocannabinoid molecule anandamide in the blood of volunteers after they ran or cycled in a lab. Because cannabis targets these same endocannabinoid receptors, Bryan speculates that the drug might allow users to "jumpstart" those pleasurable feelings.

She stresses that direct evidence connecting cannabis to runner's high remains to be found. But nevertheless, she says, people say they enjoy exercise with cannabis, which could create a positive feedback loop that motivates them to go back to the gym. "If something feels good", she says, "you're going to want to do it again."

Another way in which cannabis could encourage exercise is by aiding recovery, Bryan says. In her survey, $77 \%$ of people who use cannabis alongside exercise said that it helps with recovery. Again, researchers haven't done controlled studies looking at cannabis and recovery. So, for now, Bryan says, "we have to kind of guess based on the mechanisms that we know."
What researchers do know is that intense physical activity puts stress on the body. It triggers a flood of chemicals known as cytokines, some of which inflame muscles, that manifests as soreness the next day. Cannabis might modulate this inflammation - but potentially in multiple conflicting ways. Bryan explains that although cannabidiol (CBD), a non-psychoactive component of cannabis, has been shown to suppress pro-inflammatory cytokines, the psychoactive part, tetrahydrocannabinol (THC), stimulates both pro- and anti-inflammatory cytokines. In other words, CBD could limit the feeling of sore muscles, whereas THC could help to both prevent and trigger the discomfort. Some studies suggest that THC can also help to manage pain ${ }^{5}$, which might also boost recovery, she says.

Bryan's team found that the survey respondents who used cannabis alongside exercise tended to be younger and male. Meanwhile, a survey, the results of which are unpublished, conducted on social media by Humboldt State University in Arcata, California, which targeted people who use cannabis with exercise, had roughly an equal number of male and female participants.

Led by Whitney Ogle, a physical therapist and cannabis researcher at Humboldt, the survey of 126 people uncovered cannabis use before all sorts of physical activity -55 activities in total, ranging from archery to waterskiing. Aside from sheer enjoyment, people in the Humboldt survey reported numerous other benefits of combining cannabis with exercise. They thought that cannabis increased their focus, concentration and mind-body awareness - something that elite athletes have also reported $^{6}$, although scientists have yet to come up with possible mechanisms for these effects.

Ogle's survey also asked participants 
something the Colorado team's survey did not: did people have negative experiences after combining cannabis and exercise? About $40 \%$ of respondents reported adverse effects, which included elevated heart rate and being too high to continue with their workout, Ogle says.

The biggest limitation of the survey by the Colorado team, Bryan says, is that it didn't include a non-user control group. The researchers polled people from states such as Colorado, California and Washington, which already have higher levels of physical activity than does the country as a whole, so it's hard to tell whether cannabis motivated people to exercise more than is typical in those states. Soon, researchers could have access to populations with a wider range of activity levels. "The good news for researchers is that states are legalizing like crazy," Bryan says. Eleven US states and the District of Columbia have approved recreational marijuana use, and 33 states allow medical marijuana.

\section{EVIDENCE VERSUS ANECDOTE}

Although survey data are valuable for designing experiments, they will not provide the evidence that researchers such as Bryan crave. Currently, anecdotes concerning cannabis and exercise far outnumber controlled studies of the relationship, but that's not to say that no one has tried.

In 2018, researchers at McMaster University in Hamilton, Canada, scoured the literature for studies of marijuana's effect on athletic performance that included a control group. Only three small studies made the cut ${ }^{7}$.

Conducted between 1975 and 1986 in people under the age of 35 , two of the studies in the review ${ }^{7}$ had participants exercise before and after smoking cannabis. The third study was purely observational. In areas such as workout times, heart rate and blood pressure, the studies mostly recorded either no difference from the control groups, or negative effects. The sole positive finding, from a study with 24 participants, was an increase in forced exhalation, or

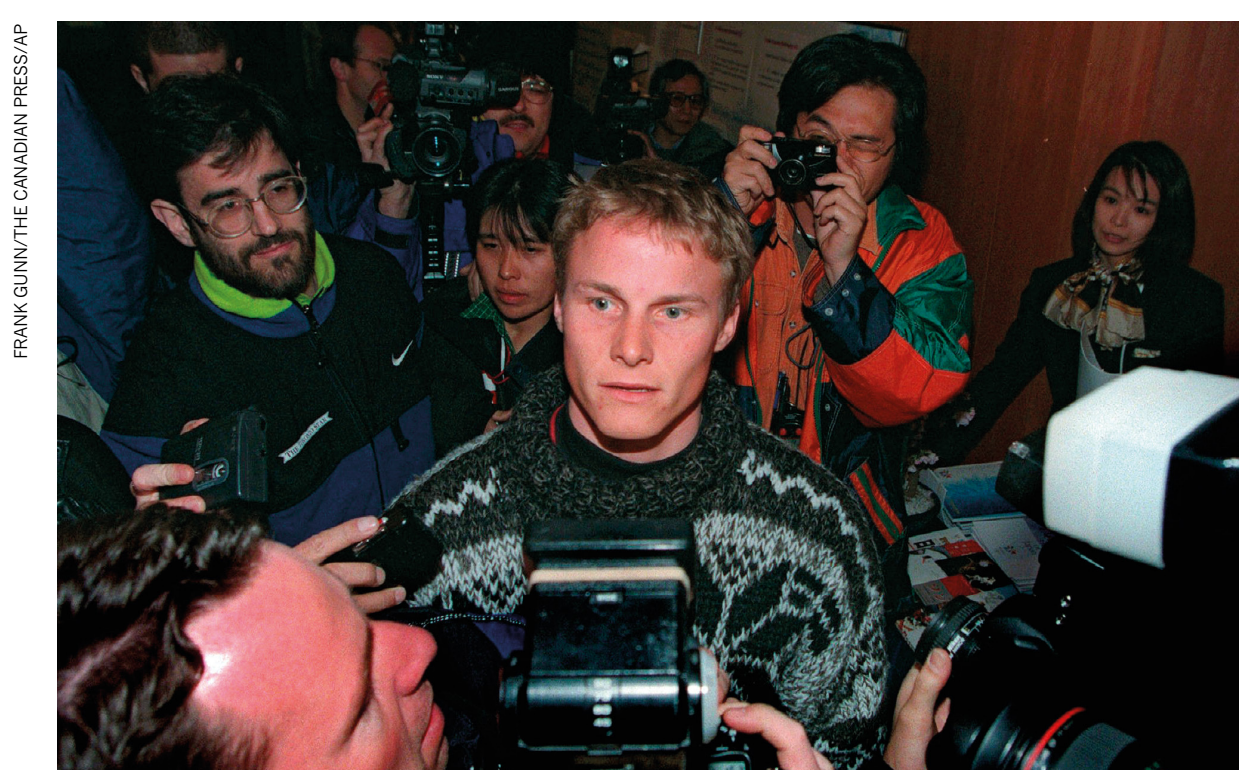

Olympic snowboarder Ross Rebagliati tested positive for the cannabinoid tetrahydrocannabinol in 1998.

the amount of air participants could exhale after taking a deep breath.

On the basis of the studies' low sample size and quality - two studies used marijuana containing about $1-2 \%$ THC, much lower than recreational levels in the United States, which in 2014 averaged around $12 \%$ - the authors concluded that the "effects of marijuana on athletic performance remain unclear".

Despite the lack of evidence that cannabis enhances performance, the concern led the World Anti-Doping Agency (WADA) to ban cannabis use during competitions when it assumed responsibility for the list of prohibited substances in 2004. According to WADA, which is based in Montreal, Canada, and is affiliated with the International Olympic Committee (IOC), the drug meets all the criteria for being banned. As well as having potential to enhance performance, it poses health risks to athletes, and is illegal in many parts of the world.

Olivier Rabin, WADA's senior science director, explained the agency's reasoning for the cannabis ban in a 2011 paper $^{8}$ co-authored by a researcher from the US National Institute on Drug Abuse. Although he acknowledges that the scientific evidence is shaky, Rabin says that the "wealth of testimonies" from athletes who admit to using cannabis to enhance their performance can't be ignored.

These accounts - mainly unpublished reports made to WADA's doping support hotlines - have come from a number of sports, he says. For example, goalkeepers in football say that cannabis increases their focus, helping them drown out the many distractions in the stadium. Athletes in other sports, such as skateboarding and skiing, say that cannabis reduces competition anxiety that can hinder performance.

Rabin says that athletes seem to be able to "titrate" their cannabis use to get just the results they want; he likens it to drinking just enough alcohol to be sociable at a party. Cannabis might not enhance performance at all doses and in all situations, he says, but its use could be advantageous in some circumstances.

Over the past several years, as perceptions of marijuana have evolved in society, so too has WADA's stance on the drug. In 1998, before WADA officially took on its anti-doping duties, Canadian snowboarder Ross Rebagliati was stripped of his Olympic gold medal after officials detected 17.8 nanograms per millilitre of THC in his system. Rebagliati's medal was reinstated soon after, on the basis that the IOC had not listed cannabis as a banned substance. In 2013, WADA raised the level of cannabis allowed in urine samples from 15 nanograms per millilitre to 150 nanograms per millilitre. Rabin says that this higher level will focus WADA's anti-doping efforts on active users of cannabis instead of infrequent users who aren't trying to enhance their performance.

Even as public acceptance of marijuana grows, researchers are finding it difficult to fully investigate cannabis's impact on physical activity because of the restrictions on cannabis research. Federal policy requires that scientists submit to an application process that can take six months to one year. On approval, researchers are only allowed to use government-provided cannabis with levels of THC that are often lower than those of cannabis available to the public. "Until regulations change," Ogle says, "we're really thwarted from being able to do really good research that we know the public wants and needs."

Researchers are coming up with creative ways to get around these rules. Some of Bryan's collaborators in Colorado have created a mobile lab - essentially, a renovated van that can be parked outside participants' homes - to test users after they have consumed their own product. Because testing takes place off-campus, scientists aren't subject to campus restrictions on cannabis. With these kinds of creative approach, Bryan says, scientists could start investigating the effects of cannabis on specific aspects of exercise such as inflammation.

But the question she'd most like to answer is probably the most difficult one: does cannabis directly influence people's decision to exercise? "To me," says Bryan, "that's the most interesting question out there."

Tien Nguyen is a freelance science journalist based in Washington DC.

\section{Le Strat, Y. \& Le Foll, B. Am. J. Epidemiol. 174 929-933 (2011).}

2. York Williams, S. L. et al. Front. Public Health 7, 1-7 (2019).

3. Gillman, A. S., Hutchison, K. E. \& Bryan, A. D. Sports Med. 45, 1357-1363 (2015).

4. Sparling, P. B. et al. NeuroReport 14, 2209-2211 (2003).

5. Borgelt, L. M., Franson, K. L., Nussbaum, A. M. \& Wang, G. S. Pharmacotherapy 33, 195-209 (2013).

6. Huestis, M. A., Mazzoni, I. \& Rabin, O. Sports Med. 41, 949-966 (2011).

7. Trinh, K. V., Diep, D. \& Robson, H. Clin. J. Sport Med. 28, 350-357 (2018).

8. Huestis, M. A., Mazzoni, I. \& Rabin, O. Sports Med. 41, 949-966 (2011). 